\title{
Effects of Thermal Stability and Incoming Boundary-Layer Flow Characteristics on Wind-Turbine Wakes: A Wind-Tunnel Study
}

\author{
Leonardo P. Chamorro • Fernando Porté-Agel
}

Received: 24 December 2009 / Accepted: 6 May 2010 / Published online: 6 June 2010

(C) Springer Science+Business Media B.V. 2010

\begin{abstract}
Wind-tunnel experiments were carried out to study turbulence statistics in the wake of a model wind turbine placed in a boundary-layer flow under both neutral and stably stratified conditions. High-resolution velocity and temperature measurements, obtained using a customized triple wire (cross-wire and cold wire) anemometer, were used to characterize the mean velocity, turbulence intensity, turbulent fluxes, and spectra at different locations in the wake. The effect of the wake on the turbulence statistics is found to extend as far as 20 rotor diameters downwind of the turbine. The velocity deficit has a nearly axisymmetric shape, which can be approximated by a Gaussian distribution and a power-law decay with distance. This decay in the near-wake region is found to be faster in the stable case. Turbulence intensity distribution is clearly non-axisymmetric due to the non-uniform distribution of the incoming velocity in the boundary layer. In the neutral case, the maximum turbulence intensity is located above the hub height, around the rotor tip location and at a distance of about 4-5.5 rotor diameters, which are common separations between wind turbines in wind farms. The enhancement of turbulence intensity is associated with strong shear and turbulent kinetic energy production in that region. In the stable case, the stronger shear in the incoming flow leads to a slightly stronger and larger region of enhanced turbulence intensity, which extends between 3 and 6 rotor diameters downwind of the turbine location. Power spectra of the streamwise and vertical velocities show a strong signature of the turbine blade tip vortices at the top tip height up to a distance of about 1-2 rotor diameters. This spectral signature is stronger in the vertical velocity component. At longer downwind distances, tip vortices are not evident and the von Kármán formulation agrees well with the measured velocity spectra.
\end{abstract}

L. P. Chamorro · F. Porté-Agel

Saint Anthony Falls Laboratory, Department of Civil Engineering, University of Minnesota, 23rd Ave. SE, Minneapolis, MN 55414, USA

F. Porté-Agel ( $\varangle)$

School of Architecture, Civil and Environmental Engineering, École Polytechnique Fédérale de Lausanne, 1015 Lausanne, Switzerland

e-mail: fernando.porte-agel@epfl.ch 
Keywords Atmospheric boundary layer - Thermal stratification - Turbulence $\cdot$ Wind-tunnel experiment · Wind-turbine wake

\section{Introduction}

Wind turbine wakes have noticeable effects on both power generation and fatigue loads in wind energy parks. Intensive numerical and experimental efforts have been carried out to better understand these effects (Vermeer et al. 2003). Wake characteristics are expected to depend on the incoming atmospheric boundary-layer (ABL) flow statistics (e.g. mean velocity distribution, turbulent stresses and turbulent fluxes). Of particular importance is the effect of velocity reduction on the mean power and turbulence intensity on the power fluctuations and fatigue loads. In particular Rosen and Sheinman (1996) showed that, for practical purposes, turbulence intensity and the intensity of turbine power fluctuations are closely related. Because turbine wakes enhance the turbulence intensity, an increment of power (and voltage) fluctuations is observed in turbines placed in wind parks. These fluctuations can affect the voltage in distribution systems. In addition, the intensity of turbulence may have an important effect on the fatigue accumulations in the structure of a wind turbine.

Atmospheric stability is also expected to have a significant impact on the characteristics of the incoming ABL flow and the structure of wind turbine wakes due to the effect of positive/negative buoyancy on the production/destruction of turbulence. Based on thermal stability (buoyancy effects) and the dominant mechanisms of turbulence generation (Stull 1988; Arya 2001), the ABL can be classified as neutral, convective or stable. Atmospheric stability and its role in wind energy is a subject of increasing research (e.g. Smedman et al. 1996; Pérez et al. 2005). In fact, field experiments performed by van der Berg (2008) have shown that atmospheric stability cannot be viewed as a small perturbation to a basic neutral state because important increments on wind shear and power production are observed in stable conditions relative to the neutral atmosphere. For example, a field study of Archer and Jacobson (2003) suggests that U.S. wind power at $80 \mathrm{~m}$ altitude may be substantially greater than previously estimated because atmospheric stability effects were not taken into account. van der Berg (2008) also showed that atmospheric stability has a significant effect on sound emissions, resulting in higher noise levels with respect to neutral stratification.

Of special interest for wind energy applications is the study of the stable boundary layer (SBL), which is common over land at night. In this flow scenario, the surface is colder than the overlaying air and turbulence is generated by shear and destroyed by negative buoyancy and viscous dissipation. Characterization and prediction of SBL flow is particularly challenging due to the occurrence of complex phenomena such as low-level jets, Kelvin-Helmholz instabilities, meandering motions, partial decoupling between the upper and bottom portions of the boundary layer and increased levels of intermittency (Hunt 1988; Derbyshire 1990; Mahrt 1998).

Wind-tunnel experiments have provided useful information about the effects of stable stratification on boundary-layer flow, and a number of laboratory studies have been carried out to better understand different aspects of the SBL. For instance, early experiments (e.g. Arya 1968; Chaudhry and Meroney 1973; Ohya et al. 1997) focused on the flow characteristics and diffusion in the SBL with different Richardson numbers. Recently, Ohya et al. (2008) investigated intermittent bursting in an SBL with a low-level jet. In spite of the different efforts to characterize the SBL, not much is known about the structure and dynamics of wind-turbine wakes in thermally stratified flows. Characterization of wind-turbine wakes under different thermal stratification conditions could provide valuable information for the optimization of 
wind turbine configurations in wind farms and the validation of parameterizations used in high-resolution computational fluid dynamic models (e.g. large-eddy simulation, LES).

In this study, results are presented from wind-tunnel experiments carried out using a model wind turbine placed inside a boundary layer under both neutral and stable thermal stratification conditions. The wind-tunnel experiments are presented in Sect. 2. Section 3 shows a comparison of the wind turbine wake characteristics, measured at different locations downwind of the turbine, for both thermal stratification regimes. Finally, a summary is given in Sect. 4.

\section{Experimental Set-up}

A 3-blade wind turbine model was placed in the thermally stratified boundary-layer wind tunnel of the Saint Anthony Falls Laboratory at the University of Minnesota with the goal of studying turbulence characteristics of the wind turbine wake. The model wind turbine was placed in a boundary layer developed over a smooth surface under neutral and stable stratified thermal conditions. The boundary-layer wind tunnel has a plan length of $37.5 \mathrm{~m}$ with a main test-section fetch of roughly $16 \mathrm{~m}$ and a cross section of $1.7 \mathrm{~m} \times 1.7 \mathrm{~m}$. There is a contraction with a $6.6: 1$ area ratio upwind of the test section along with flow conditioning/turbulence control consisting of a coarse wire mesh and honeycomb flow-straightener. The tunnel is driven by a 200 horse-power fan and is operated as a closed return loop. Turbulence intensity in the centre (free stream) of the wind tunnel is approximately $1 \%$ for a $2.5 \mathrm{~m} \mathrm{~s}^{-1}$ free stream velocity. More details on the wind tunnel can be found in Carper and Porté-Agel (2008).

The test-section floor consisted of heated exchangers made of aluminum plates $0.3 \mathrm{~m}$ long and $25.4 \mathrm{~mm}$ thick through which a solution of $30 \%$ ethylene glycol was circulated. In addition, a heat exchanger was used to control the temperature of the air in the wind-tunnel expansion after the fan. Electronic valves were used to automatically control (using a Labview code) the required temperatures of both the air flow and the test section floor with an accuracy of $\pm 0.2^{\circ} \mathrm{C}$.

A turbulent boundary layer was developed with the help of a tripping mechanism $(40 \mathrm{~mm}$ picket fence) located at the exit of the wind-tunnel contraction, where the test section begins. The turbulent boundary layer was allowed to grow in zero pressure gradient conditions by adjustment of the wind-tunnel ceiling. The resulting boundary layer has a well-developed surface layer with constant shear stress and a logarithmic velocity profile for the neutral stratification regime (see Chamorro and Porté-Agel 2009 for more details).

The experiments were conducted with a free stream velocity of approximately $2.5 \mathrm{~m} \mathrm{~s}^{-1}$. A turbulent boundary-layer depth of $\delta \approx 0.5 \mathrm{~m}$ was obtained at the turbine location for the two stratification cases (neutral and stable). The zero pressure gradient boundary layer had a Reynolds number, based on the boundary-layer depth $(\delta)$, of $R e_{\delta}=U_{\infty} \delta / v \approx 0.93 \times 10^{5}$, and a friction velocity of $u_{*}=0.11 \mathrm{~m} \mathrm{~s}^{-1}$. The aerodynamic roughness length was found to be $z_{0}=0.05 \mathrm{~mm}$. These parameters $\left(u_{*}\right.$ and $\left.z_{0}\right)$ were obtained by fitting a logarithmic velocity profile to the measured average velocity in the surface layer (approximately the lowest $15 \%$ of the boundary layer).

The stable stratified boundary-layer case was created by cooling the floor to $7^{\circ} \mathrm{C}$ and heating the wind-tunnel air flow to $60^{\circ} \mathrm{C}$. Based on these conditions, the SBL had a bulk Richardson number $R i_{\delta}\left(=g \delta \Delta T /\left(T_{0} U_{\infty}^{2}\right)\right) \approx 0.15$, which corresponds to weak stability. Here $g$ is the acceleration due to gravity, $\Delta T$ is the temperature difference (in $\mathrm{K}$ ) between the free stream flow and the surface, $T_{0}$ is a reference temperature (in $\mathrm{K}$ ) and $U_{\infty}$ is the free stream velocity. Figure 1 shows the vertical profiles of the mean velocity, mean temperature, 
turbulence intensity, kinematic shear stress and kinematic heat flux measured in the boundary layer with stable stratification.

Mean wind velocity in the tunnel free stream was measured using Pitot static tubes (mainly for calibration purposes) and a customized 3-wire anemometer. The sensor (a combination of an x-type hot-wire and a single cold-wire) was used to obtain high resolution and simultaneous measurements of the two velocity components (streamwise and vertical) and temperature. The probe was made of $5.0 \mu \mathrm{m}$ tungsten wires (used as hot wires for velocity measurements) and a $2.5 \mu \mathrm{m}$ wire (used as cold wire for temperature measurements), which are connected to an A.A. Lab Systems AN-1003 10-channel CTA/CCA system (for details see Vukoslavcevic and Wallace 2002). During the calibration and measurements the temperature fluctuations were kept within a $\pm 0.2^{\circ} \mathrm{C}$ range to avoid bias errors due to thermal drift of the voltage signal.

Calibration of the sensor was performed at the beginning of each experimental run. The 3-wire anemometer was calibrated in the free stream region, against a Pitot-static probe (for mean velocity) and a thermocouple (for mean temperature), considering seven sensor inclination angles and seven wind velocities at each position. The process was repeated for four different mean free stream temperatures. A combination of cubic-spline table calibration methods was then used to determine the two instantaneous velocity components and temperature. For more details on the calibration procedure, see Bruun (1995).

The model wind turbine consisted of a three-blade GWS/EP-6030x3 rotor attached to a small DC generator. The turbine angular velocity was adjusted by changing the resistance of the generator. During the experiments, the tip speed ratio was approximately $\lambda=$ $2 \pi r \Omega /\left[60 U_{h u b}\right] \approx 4$, where $\Omega$ is the angular velocity of the turbine in r.p.m. and $U_{h u b} \approx$ $2.1 \mathrm{~m} \mathrm{~s}^{-1}$ is the mean velocity at the hub height. A similar turbine was used by Chamorro and Porté-Agel (2009) to study roughness effects on the turbulent properties of the wake under neutral conditions.

The tip speed ratio was adjusted to match that of field-scale turbines (usually between 3.5 and 6). As shown in Fig. 1, the bottom tip of the turbine was set to a height of 0.67 times the turbine radius, which is similar to that found in large-scale turbines ( $\geq 2 \mathrm{MW})$. The turbine rotor was roughly within the lowest third of the turbulent boundary layer. Despite the scaling issues associated with the difference in Reynolds number between the wind-tunnel
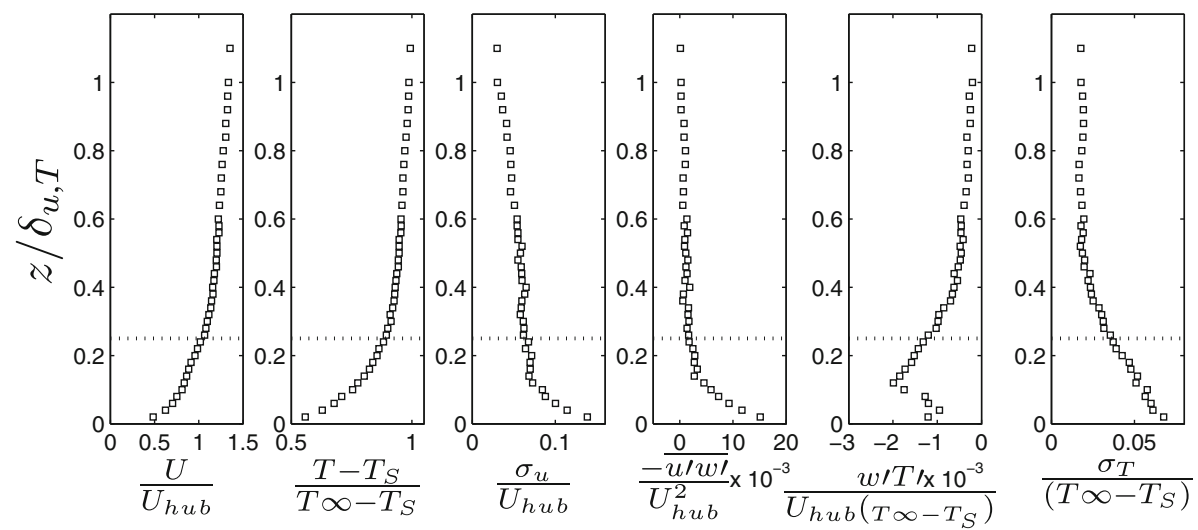

Fig. 1 Background characteristics of the stable boundary layer: normalized mean velocity, mean temperature, turbulence intensity, kinematic shear stress and kinematic heat flux. The horizontal lines represent the turbine axis. Height is normalized with the turbine radius $r$ 
flow and the ABL flow, our measurements provide detailed key information about the qualitative behaviour of turbine wakes in turbulent boundary-layer flows. It is also important to note that the high-resolution spatial and temporal measurements presented here can be used to systematically test the performance of numerical models (e.g. LES with different subgrid-scale models and wind turbine force parameterizations) in the simulation of wind turbine wakes in turbulent boundary-layer flows. Okulov and Sorensen (2007) have shown also that, although it is not possible to match the Reynolds number of real wind turbines, it is possible to reproduce the basic characteristics of the wakes (e.g. wake rotation, tip vortices and helicoidal vortices).

As shown in Fig. 2, the 3-wire anemometer was placed at different positions downwind of the turbine $(x / d=2,3, \ldots, 10,12, \ldots, 20$; where $d$ is the turbine diameter). At each location, measurements were taken at zero $\operatorname{span}(y=0)$ for elevations ranging from $z=10 \mathrm{~mm}$ to $z=300 \mathrm{~mm}$ every $\Delta z=10 \mathrm{~mm}$. In addition, a cross-sectional characterization of the wake was obtained at $x / d=5$. There, measurements were taken in a plane that spans from $z=0$ to $0.3 \mathrm{~m}$ in the vertical direction and from $y / r=-1.60$ to 1.33 in the spanwise direction. On that plane, measurements were taken over a grid of points separated by $\Delta y=\Delta z=15 \mathrm{~mm}$. The sensor voltage signatures (of two velocity components and temperature) were sampled at rates of 0.5 and $1 \mathrm{kHz}$ for a measurement period ranging from 30 to $120 \mathrm{~s}$.

\section{Wake Properties}

In this section we present flow statistics at different locations downwind of the turbine $(x / d=2,3, \ldots, 10,12, \ldots, 20)$ at zero span (see Fig. 2$)$ for the two thermal stratification cases (neutral and stable). A cross-sectional characterization of the turbine wake at $x / d=5$ is also included. Particular emphasis is placed on the distribution of the non-dimensional distribution of the normalized mean velocity, $U / U_{h u b}$ (where $U_{h u b}$ is the mean velocity at the turbine hub height), turbulence intensity, $I_{u}=\sigma_{u} / U_{h u b}$, and kinematic shear stress, $\overline{u^{\prime} w^{\prime}} / U_{h u b}^{2}$, for the two stratification cases.

Figure 3 shows the distribution of the normalized mean velocity, $U / U_{h u b}$, on a vertical plane at zero $\operatorname{span}\left(\left.(x, z)\right|_{y=0}\right)$ for the two stratification cases. In both cases, the momentum loss is evident even at a distance of 20 rotor diameters downwind of the turbine. This figure also reveals clear differences in the mean velocity distribution inside the turbine wake between both thermal stratification cases. In particular, the velocity deficit in the near-wake region appears to be larger in the case of neutral stratification. These differences are associated with the non-uniform velocity distribution of the incoming boundary-layer flow, which is affected by thermal stratification. This is also the reason for the non-axisymmetric distribution of the velocity inside the wake, as illustrated in the mean velocity profiles shown Fig. 4a for the neutral case.

As pointed out by Chamorro and Porté-Agel (2009), the velocity deficit ( $\Delta U_{x}=U(x>$ $0, y, z)-U(x<0, y, z))$, shown in Fig. $4 \mathrm{~b}$, has a nearly axisymmetric shape. Note that in a fully developed boundary layer the incoming flow does not depend on streamwise and spanwise positions $x$ and $y$, and therefore $U(x<0, y, z)$ can be written as simply $U_{o}(z)$. The overall velocity deficit distribution $\Delta U_{x}\left(=U-U_{o}\right)$ in a plane perpendicular to the turbine axis (at different downwind locations $x$ in the wake) can be roughly approximated by a Gaussian distribution (Fig. 4b). This property allows us, in principle, to extend simple wake velocity formulations, developed for nearly homogeneous inflow conditions and based on similarity arguments (e.g. Frandsen et al. 2006), to more complex boundary-layer cases by modelling the velocity deficit in the wake as 

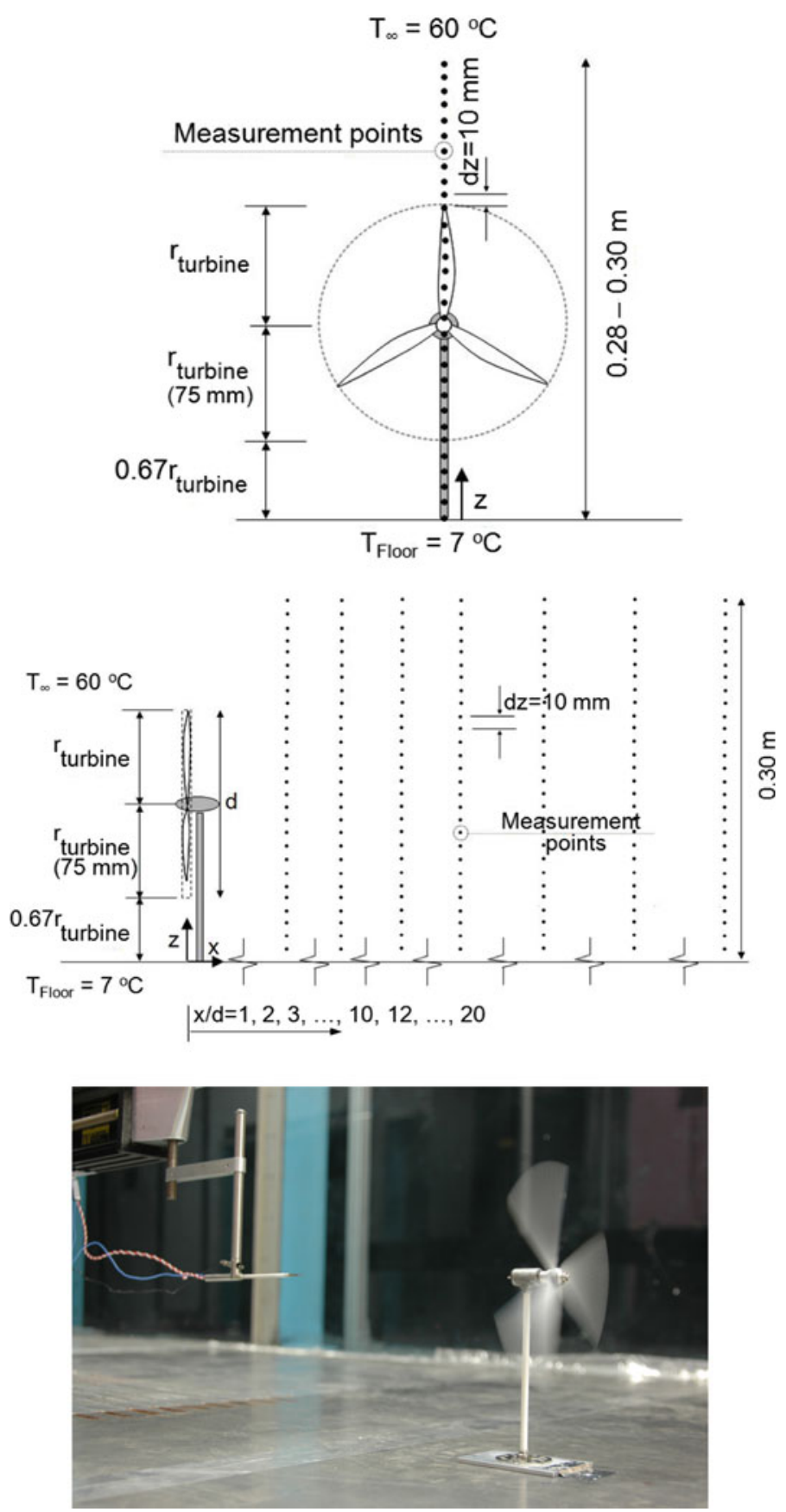

Fig. 2 Schematic of the wind turbine (front and side views), coordinate system and measurement locations (top and middle), and photograph of the test section with the wind turbine (bottom) 

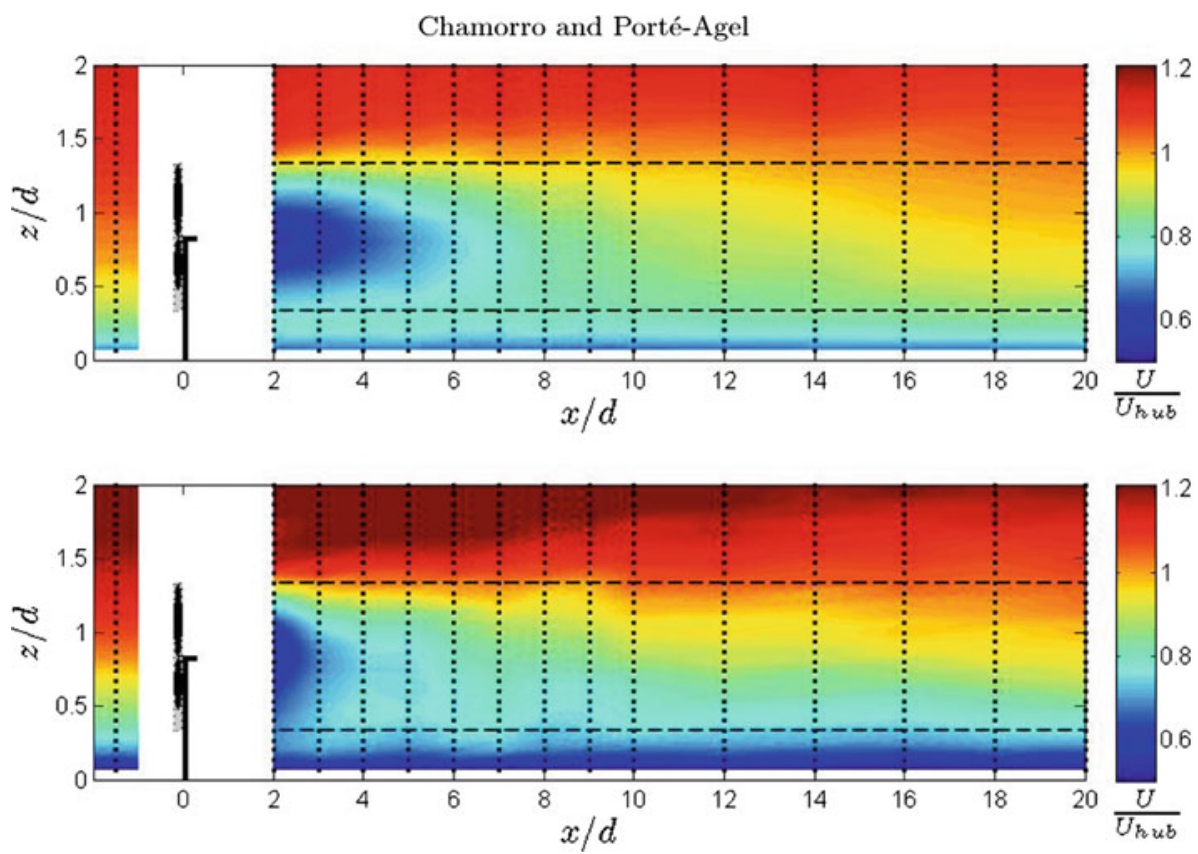

Fig. 3 Non-dimensional distribution of mean velocity downwind of the turbine at zero span in the neutral (top) and in the stable stratified (bottom) boundary layers. Horizontal-dashed lines represent the turbine bottom and top tip heights and dots indicate measurement locations

$$
\Delta U_{x}(x, r)=\Delta U_{h u b}(x) f(r / R),
$$

where $\Delta U_{h u b}(x)$ is the maximum velocity deficit in the wake (approximately at the hub height), $r$ is the distance from the centre of the wake and $R$ is a characteristic width of the wake at distance $x$ downwind of the rotor. Equation(1) suggests that the velocity deficit at the centre of the wake $\Delta U_{h u b}(x)$ is key to the characterization of the overall wake. Because it modulates the magnitude of the velocity deficit throughout the wake, its adequate prediction is essential.

Different expressions have been proposed for estimating the mean velocity at hub height, $U_{h u b}$, or its deficit, $\Delta U_{h u b}$. Some of those simple approaches are based on field data fitting (e.g. Barthelmie et al. 2004), while others consider a top-hat wake shape, i.e. a uniform velocity distribution inside the wake (e.g. Jensen 1983).

The formulation proposed by Barthelmie et al. (2004) is based on fitting field measurements in the wake at the hub height, viz.

$$
\frac{\Delta U_{h u b}(x)}{U_{\infty}}=a\left(\frac{x}{d}\right)^{b}
$$

where $U_{\infty}$ is the freestream wind speed and coefficients $(a, b)$ with values of $(1.03,-0.97)$ or $(1.07,-1.11)$ are equally plausible.

A well-known model for wake velocity with a top-hat shape is the Risoe WAsP model (Jensen 1983; Katic 1986) and, based on a linear expansion of the wake, states:

$$
U_{\text {wake }}=U_{\infty}\left[1-\left(1-\sqrt{1-C_{T}}\right)\left(\frac{d}{d+2 k_{\text {wake }} x}\right)^{2}\right] \text {, }
$$



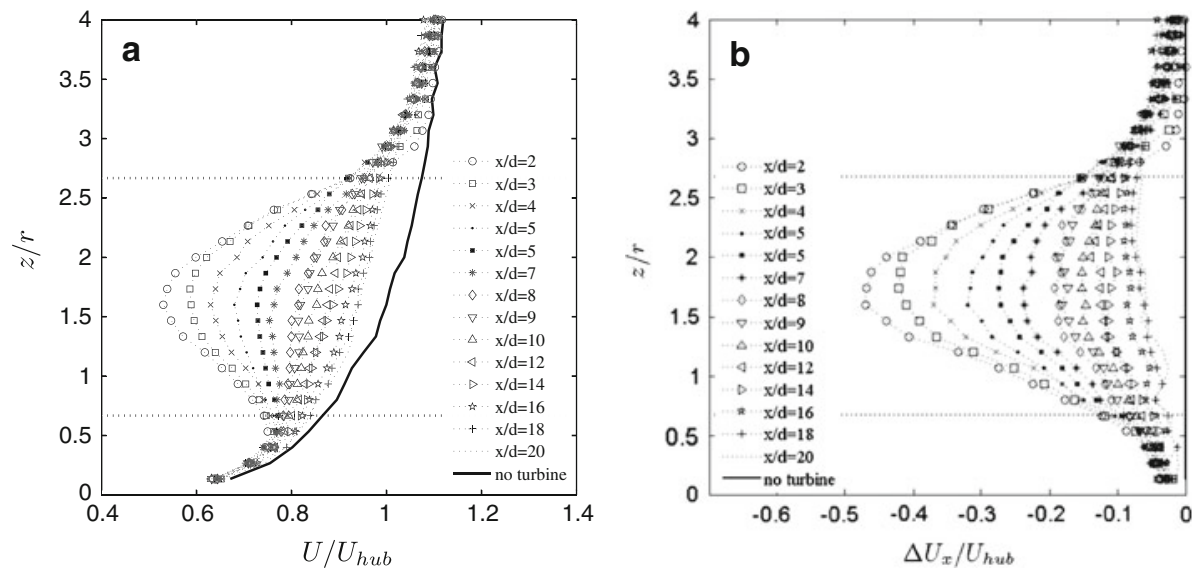

Fig. 4 Non-dimensional distribution of mean velocity (left plot) and velocity deficit (right plot) downwind of the turbine at zero span. Turbine in the neutral boundary layer. Horizontal-dotted lines represent the turbine bottom and top tip heights

where $C_{T}$ is the thrust coefficient, $k_{\text {wake }}=A / \ln \left(h_{h u b} / z_{0}\right)$ is a wake decay constant, $A \approx$ $0.5, z_{0}$ is the surface roughness length and $x$ is the distance from the turbine.

Figure 5 shows the change of normalized mean velocity, at turbine hub height, as a function of normalized downwind distance for the two stratification cases. There, a comparison with the models given in Eqs. 2 and 3 is included. The figure reveals a non-negligible departure between the model proposed by Barthelmie et al. (2004) and the measurements at distances $x / d<12$. Beyond that location the differences are small. The overall comparison of the measurements with the model suggests that the structure of the mean velocity deficit cannot be adequately represented using a power-law decay with exponent $\approx-1$. Actually, as indicated by Schlichting (1968), a velocity deficit $\Delta U_{x} \propto x^{-1}$ holds for a wake developed in a laminar flow (or where diffusion is controlled by a constant ambient turbulent diffusivity). On the other hand, the Risoe WAsP model (Eq. 3) clearly underestimates the velocity deficit at all distances. This is expected since, as mentioned above, the model assumes a uniform velocity distribution in the wake, which leads to the observed underestimation at the centre of the wake (where the maximum velocity deficit is found). In general, the velocity deficit decays relatively fast up to a distance of roughly $x / d=10$, and beyond that location, the velocity recovery is markedly slower. From an engineering perspective this implies that the incremental power $(\Delta P)$ gained by moving a second turbine, placed in the wake of another turbine, an incremental downwind displacement $(\Delta x)$ is much larger at $x / d \leq 10$. In particular, it affects the ratio power/area, which is one of the parameters that has to be maximized in wind farm design.

Figure 5 also shows that, at distances $x / d<10$, the velocity recovery appears to be faster in the stable boundary-layer case. Beyond that location, the velocity recovery appears to be similar in both stratification cases. These results agree with field measurements of Magnusson and Smedman (1999) showing that, for $R i>0.25$, there is a tendency for the velocity deficit to decrease, which would indicate more effective mixing in the wake.

The overall velocity recovery throughout the wake is strongly related to radial transport. In order to better understand this complex relation, we consider the continuity equation in cylindrical coordinates: 


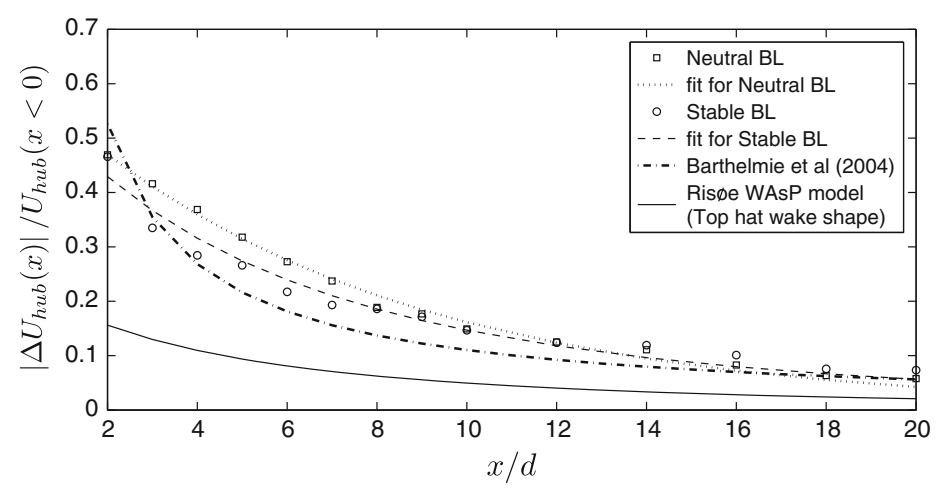

Fig. 5 Normalized velocity deficit at hub height for the two stratification cases and its comparison with simple models

$$
\frac{1}{r} \frac{\partial}{\partial r}\left(r U_{r}\right)+\frac{1}{r} \frac{\partial}{\partial \theta}\left(U_{\theta}\right)+\frac{\partial U_{x}}{\partial x}=0
$$

where a schematic of the positioning of the cylindrical coordinate system is shown in Fig. 6. The second term in Eq. 4 is presumably much smaller than the others due to an expected low dependence of the tangential velocity on angle and therefore, for a first-order analysis, it can be neglected. From Fig. 4 one notes that the streamwise velocity gradient is clearly positive between (roughly) bottom and top tip heights and negative above the turbine top tip. For the region where $\partial U_{x} / \partial x>0$, necessarily:

$$
\frac{\partial}{\partial r}\left(r U_{r}\right)<0 \Leftrightarrow-U_{r} / r>\partial U_{r} / \partial r
$$

The left part of Eq. 5 implies that, for $r>0, U_{r}$ is negative and the right part implies a convex shape of $U_{r}$ with normal distance $r$. Similarly, above the top tip $\partial U_{r} / \partial r<0$ and $-U_{r} / r<\partial U_{r} / \partial r$, suggesting a concave shape of $U_{r}$ (with $U_{r}$ approaching zero as $r$ increases). The intersecting point around the top tip height, where the streamwise velocity gradient is nearly zero, is an inflexion point for the radial velocity component. The behaviour of $U_{r}$ with the positive and decreasing streamwise velocity gradient $\partial U_{x} / \partial x$ modulates the radial transport through the wake downwind of the turbine. From this it is inferred that, at the very far wake where $\partial U_{x} / \partial x \approx 0$, the radial transport is low.

In the so-called very far wake region $(x / d \geq 10)$ the streamwise velocity gradient is markedly smaller, leading to a small radial velocity and a reduced wake growth. Mixing of the wake with the surrounding flow is responsible for the reduced streamwise velocity gradient.

Figure 7 shows the turbulence intensity $\left(\sigma_{u} / U_{h u b}\right)$ on a vertical plane at zero span $\left(\left.(x, z)\right|_{y=0}\right)$ for both thermal stratification conditions. A strong enhancement of turbulence intensity is found in the wake around the top-tip height. The enhancement of turbulence intensity is associated with strong shear and turbulence kinetic energy production in that region. Due to boundary-layer effects (relatively higher incoming turbulence intensity and shear), negligible effects are observed near the bottom tip height, where the mean shear is smaller (Chamorro and Porté-Agel 2009). In addition to the boundary-layer effects, negative buoyancy acting in the stable case precludes an enhancement of turbulence intensity in the lower part of the wake. 
Fig. 6 Schematic of the cylindrical coordinate system and velocity components
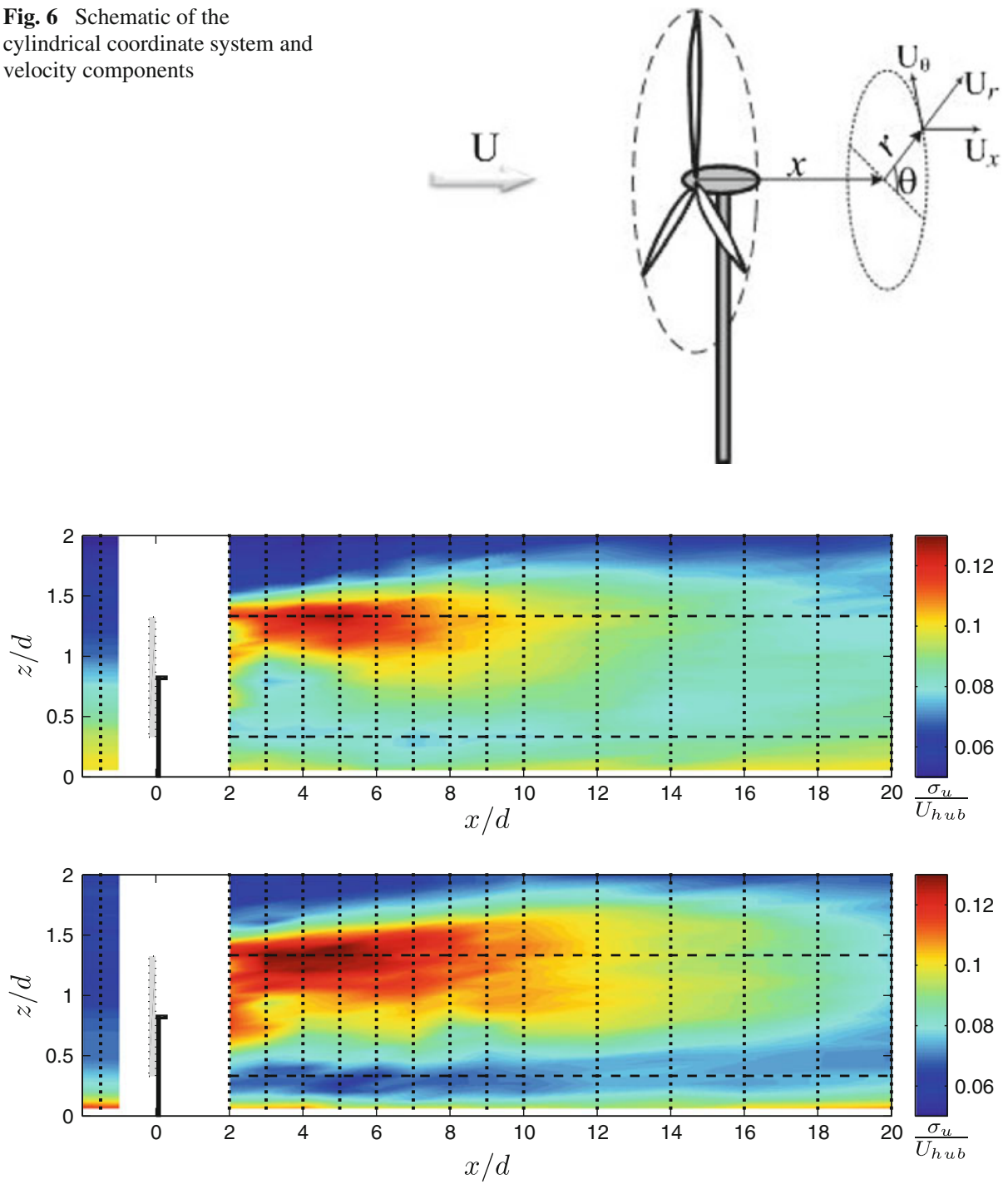

Fig. 7 Turbulence intensity distribution downwind of the turbine at zero span in the neutral (top) and in the stable stratified (bottom) boundary layer. Horizontal-dashed lines represent the turbine bottom and top tip heights and dots indicate measurement locations

The region of higher turbulence intensity (above the hub level and near the turbine tip height) extends up to approximately $x / d \approx 8$. Its peak is located between distances of roughly 4-5.5 turbine diameters for the neutral case and between 3 and 6 turbine diameters in the stable situation. In addition, the maximum turbulence intensity appears to be larger in the stable case, consistent with the stronger mean shear (and turbulent kinetic energy production) at top tip level in the stable case. This potentially implies stronger turbulent loads and power fluctuations in wind turbines placed inside the wake of another turbine. In fact, an inter-turbine separation of 5 turbine diameters is common practice in wind energy parks. 

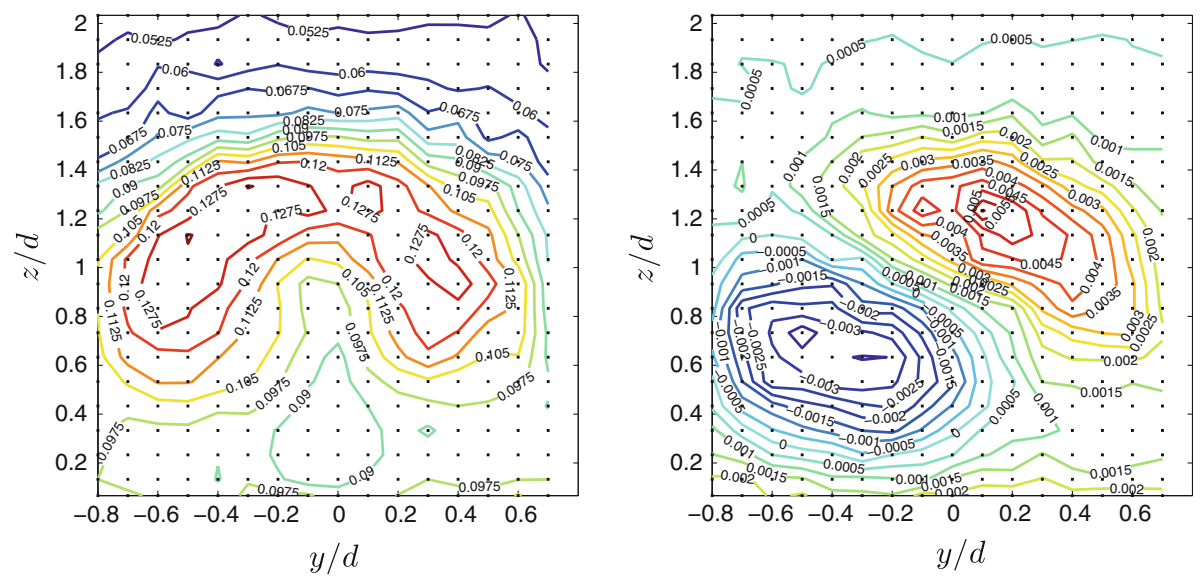

Fig. 8 Contours of turbulence intensity, $\sigma_{u} / U_{h u b}$, (left) and non-dimensional kinematic shear stress, $-\overline{u^{\prime} w^{\prime}} / U_{h u b}^{2},($ right $)$ distributions in the neutral boundary layer at $x / d=5$. Dots indicate measurement locations

Figure 7 shows also that, in the neutral case, the turbulence intensity profile is roughly uniform in the very far wake region $(x / d>10)$. The uniformity remains even at a distance of $x / d=20$, implying that not only is the mean velocity not fully recovered at that location, but also the level of turbulence fluctuations. In the stable case, turbulence intensity does not reach a homogeneous profile at any location and, below the bottom tip height, appears to be consistently smaller than at higher locations.

The non-axysimmetric character of the turbulence intensity distribution is illustrated in the cross-sectional plane at $x / d=5$ shown in Fig. 8. There, an enhancement is observed above the hub height, with maximum values around the turbine tip. Some departure from $z$-axis symmetry (at zero span) is also observed due to the rotation of the wake. The slight departure from symmetry ( $z$-axis) is characterized by a small offset from its zero span axis, which implies a difference in turbulence intensity magnitude on the two sides of the $z$-axis of the turbine, specifically $I_{u}\left(-y_{0} / d, z_{0} / d\right)>I_{u}\left(y_{0} / d, z_{0} / d\right)$ where $\left(y_{0}, z_{0}\right)$ is a location in the wake below the top tip height.

Figure 9 shows the distribution of the normalized turbulent shear stress on a vertical plane at zero span $\left(\left.(x, z)\right|_{y=0}\right)$ for both thermal stratification conditions. The mixing effect of the wake produces a large positive turbulent stress (downward momentum flux) above the hub height and a negative turbulent stress (upward momentum flux) below the hub height. The positive flux is larger in magnitude and extends to longer downwind distances due to the relatively larger shear found at the top tip level (compared with the bottom tip level) as shown in Fig. 4. From Fig. 9, some differences are also found in the near-wake distribution of the turbulent shear stress between the two stratification conditions. Specifically, in the stable case the magnitude of the positive shear stress is smaller and that of the negative shear stress is larger, compared with the neutral case. In the far wake region $(x / d>10)$, the turbine wake is dominated by roughly negligible shear, in contrast with the neutral case, which shows positive values throughout the vertical profile in that region. It is also important to point out that, as shown in Fig. 8, the spanwise distribution of the turbulent stress has a strong departure from $z$-axis symmetry (at zero span) due to the rotation of the wake. As reported by Chamorro and Porté-Agel (2009), the maximum values (positive and negative) of the stress are not located 

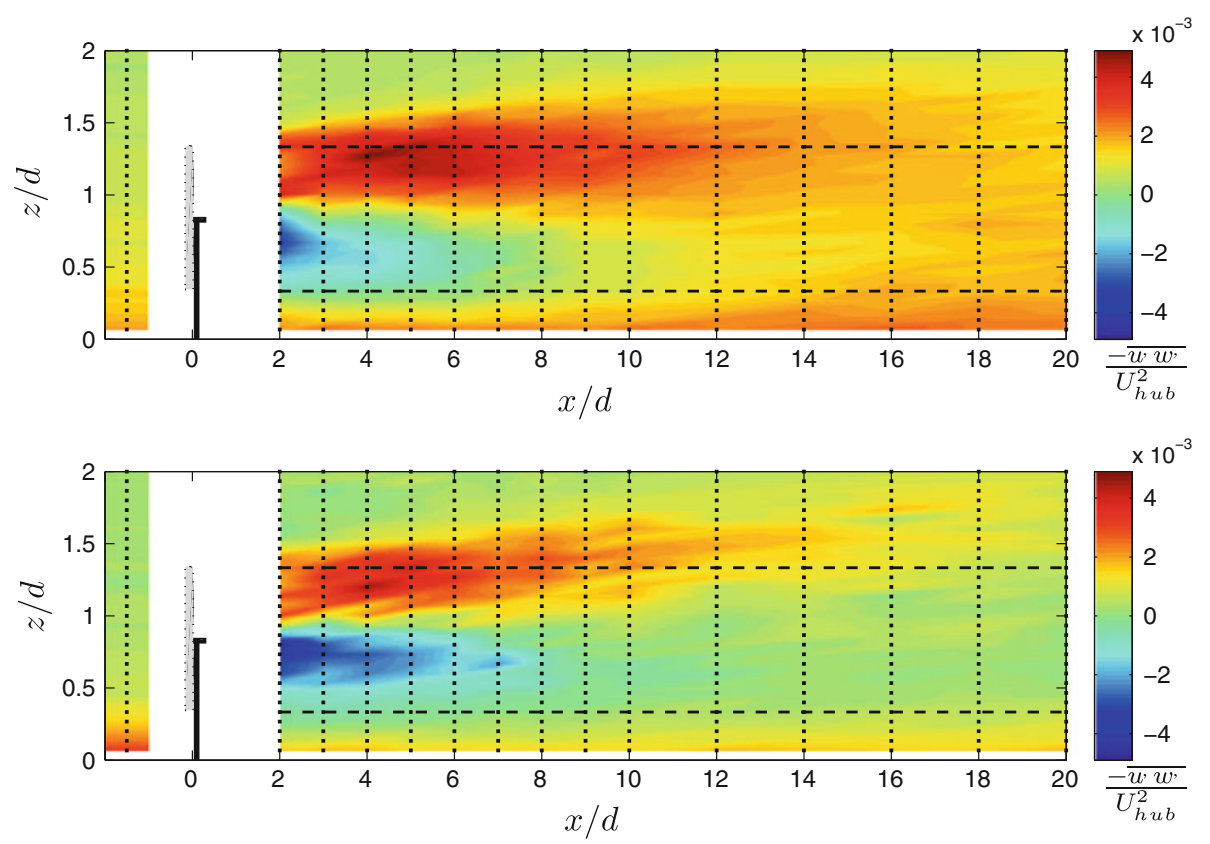

Fig. 9 Non-dimensional distribution of kinematic shear stress downwind of the turbine at zero span in the neutral (top) and in the stable stratified (bottom) boundary layer. Horizontal-dashed lines represent the turbine bottom and top tip heights and dots indicate measurement locations

on the vertical plane at zero span $\left.\left.(x, z)\right|_{y=0}\right)$, but on the rotated axis of symmetry and at a radial distance similar to the turbine tip position (radius of the turbine).

In order to further understand the effect of the turbine on the level of turbulence in its wake, Fig. 10 shows the spatial distribution of the turbulence energy production $\left(-\overline{u^{\prime} w^{\prime}} \partial U / \partial z\right.$ component). Turbulence production is largest in the upper half of the wake (above the hub height) and extends up to a distance of roughly $x / d \approx 8$. The maximum energy production level coincides approximately with the turbine top tip height and it extends between about 3-5 rotor diameters. This is consistent with the maximum enhancement of the turbulence intensity reported in Fig. 7. Similarly, the low energy production level at the bottom tip heights leads to the relatively small turbulence intensity enhancement in that region.

The power spectral density of the streamwise and vertical velocity components, shown in Fig. 11 at $x / d=1$ and zero span, reveals the strong signature of the helicoidal tip vortices shed by the turbine blades (peak of the spectrum at a frequency coincident with three times the frequency of the turbine). It is important to note that the tip vortices leave a stronger signature in the vertical velocity spectrum, compared with the streamwise velocity spectrum. At $x / d=1$ the effect of the tip vortices on the spectrum is observed up to a height of $0.27 r$ (where $r$ is the turbine radius) above the turbine top tip. The frequency associated with these tip vortices falls within the inertial subrange (spectral slope $\beta=-5 / 3$ ). As pointed out by Chamorro and Porté-Agel (2009), the relatively high turbulence levels of the incoming flow precludes a noticeable effect of the tip vortices at the bottom tip height (result not shown here).

Next, the applicability of the von Kármán spectrum (von Kármán 1948) to represent the structure of the turbulence in the wake is tested using velocity measurements collected at a 

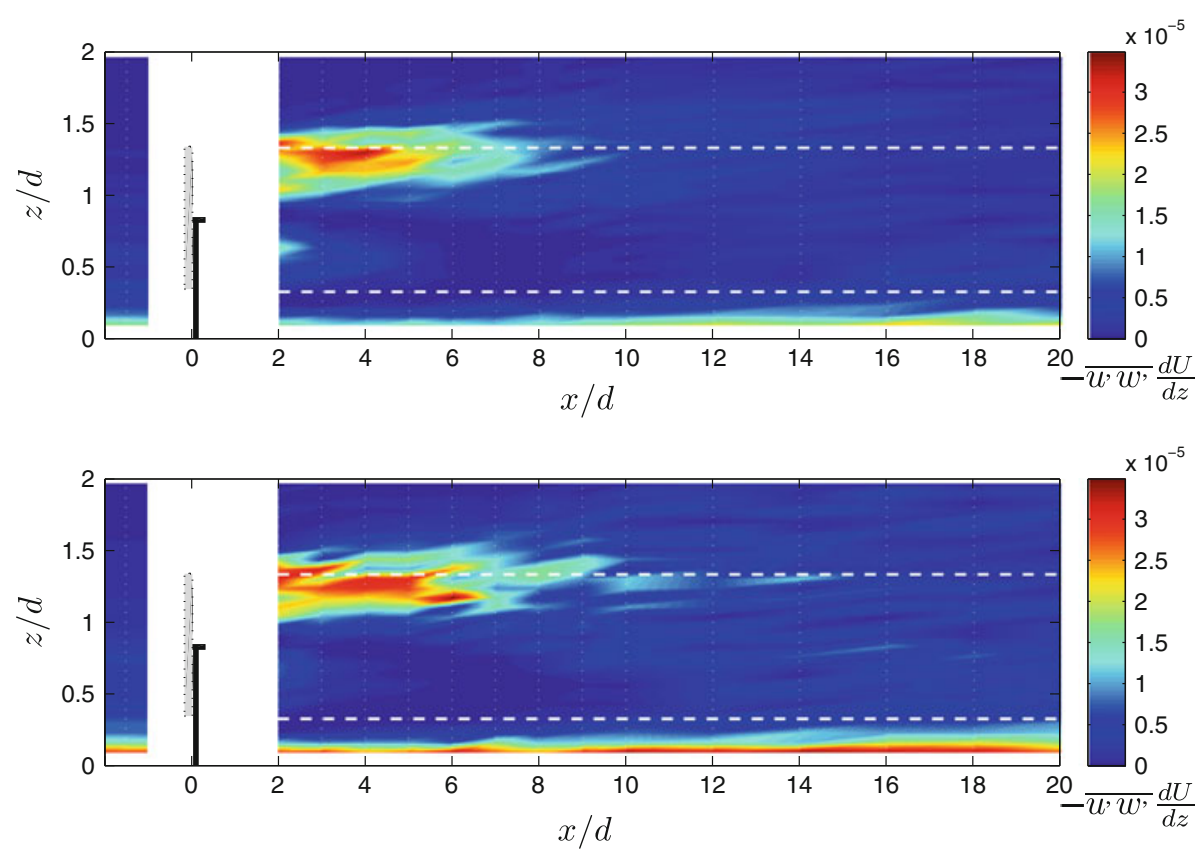

Fig. 10 Turbulent energy production $\left(-\overline{u^{\prime} w^{\prime}} d U / d z\right.$ component) at different positions downwind of the turbine (at zero span) in the neutral boundary layer (top) and in the stable boundary layer (bottom). Horizontal-dashed lines represent the turbine axes

height coincident with the turbine top tip. The von Kármán spectral density formulation for the streamwise velocity component, $S_{u}(f)$, and for the vertical velocity component, $S_{w}(f)$, are given by:

$$
\begin{aligned}
\frac{f S_{u}(f)}{\sigma_{u}^{2}} & =\frac{4 n_{u}}{\left(1+70.8 n_{u}^{2}\right)^{5 / 6}}, \\
\frac{f S_{w}(f)}{\sigma_{w}^{2}} & =\frac{4 n_{w}\left(1+755.2 n_{w}^{2}\right)}{\left(1+283.2 n_{w}^{2}\right)^{11 / 6}},
\end{aligned}
$$

where $f$ is the frequency, $\sigma_{u}^{2}$ and $\sigma_{w}^{2}$ are the variances of the streamwise and vertical velocity components, $n_{i}=f L_{i} / \overline{U_{i}}$, and $L_{i}$ and $\overline{U_{i}}$ are the $i$ th components of the integral length scale of turbulence (which can be obtained from the autocorrelation function) and mean velocity, respectively.

Figure 12 shows the comparison between measured and modelled power spectra of the streamwise and vertical velocity components, respectively, at $x / d=1$ and 5 behind the turbine, and also in the absence of the turbine. Morfiadakis et al. (1996), using field measurements, found that the von Kármán spectrum is suitable for free stream conditions (no turbine), but intense topography and wake effects were not adequately modelled by this formulation. Our results show that the von Kármán spectrum is able to represent the structure of the turbulence behind the wake in a boundary layer developed over a smooth flat surface (i.e. no significant topographic effects) in the regions where tip vortices have no strong effect on the flow. In fact Fig. 12 shows good agreement between both spectra (measured and modelled) 

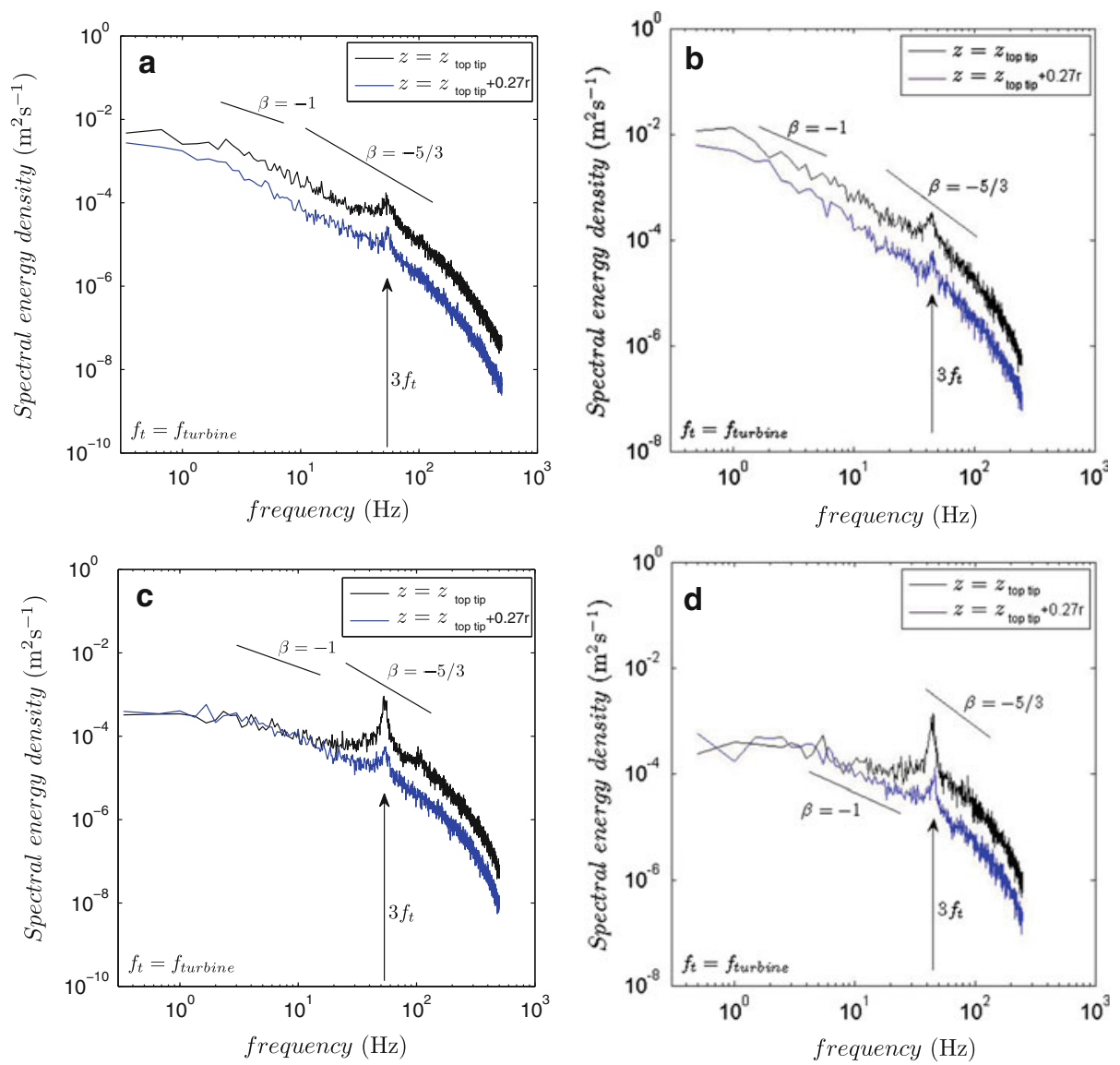

Fig. 11 Power spectrum of velocity (streamwise and vertical components) at $x / d=1$ in the neutral and stable boundary layers. a Streamwise velocity spectrum in the neutral boundary layer, b streamwise velocity spectrum in the stable boundary layer, c vertical velocity spectrum in the neutral boundary layer, $\mathbf{d}$ vertical velocity spectrum in the stable boundary layer

except at $x / d=1$, where tip vortices have an intense effect on the structure of the turbulence in a narrow range of frequencies around the blade rotation frequency.

Kinematic heat flux and mean temperature distributions, in the stable boundary-layer case, are affected by the rotating and turbulent nature of the wake. As shown in Fig. 13, the kinematic heat-flux distribution $\left(\overline{w^{\prime} \theta^{\prime}}\right)$ is strongly affected by the wake in the near-wake region $(x / d<5)$. In that region, the turbulent heat flux is relatively small. In contrast, the wake has a relatively minor effect on the distribution of the kinematic heat flux in the far-wake region.

Figure 14 shows the mean temperature distribution between the bottom and top tip heights at different distances downwind of the turbine. An increment of temperature above the hub height is evident between distances of 2 and 8 turbine diameters. At longer distances this effect is negligible, i.e. there is no relevant change in the vertical distribution of temperature with distance. Around the bottom tip height, an increase in temperature is observed at the same distances, which is consistent with the relatively large magnitude of the negative vertical turbulent heat flux in that region. 

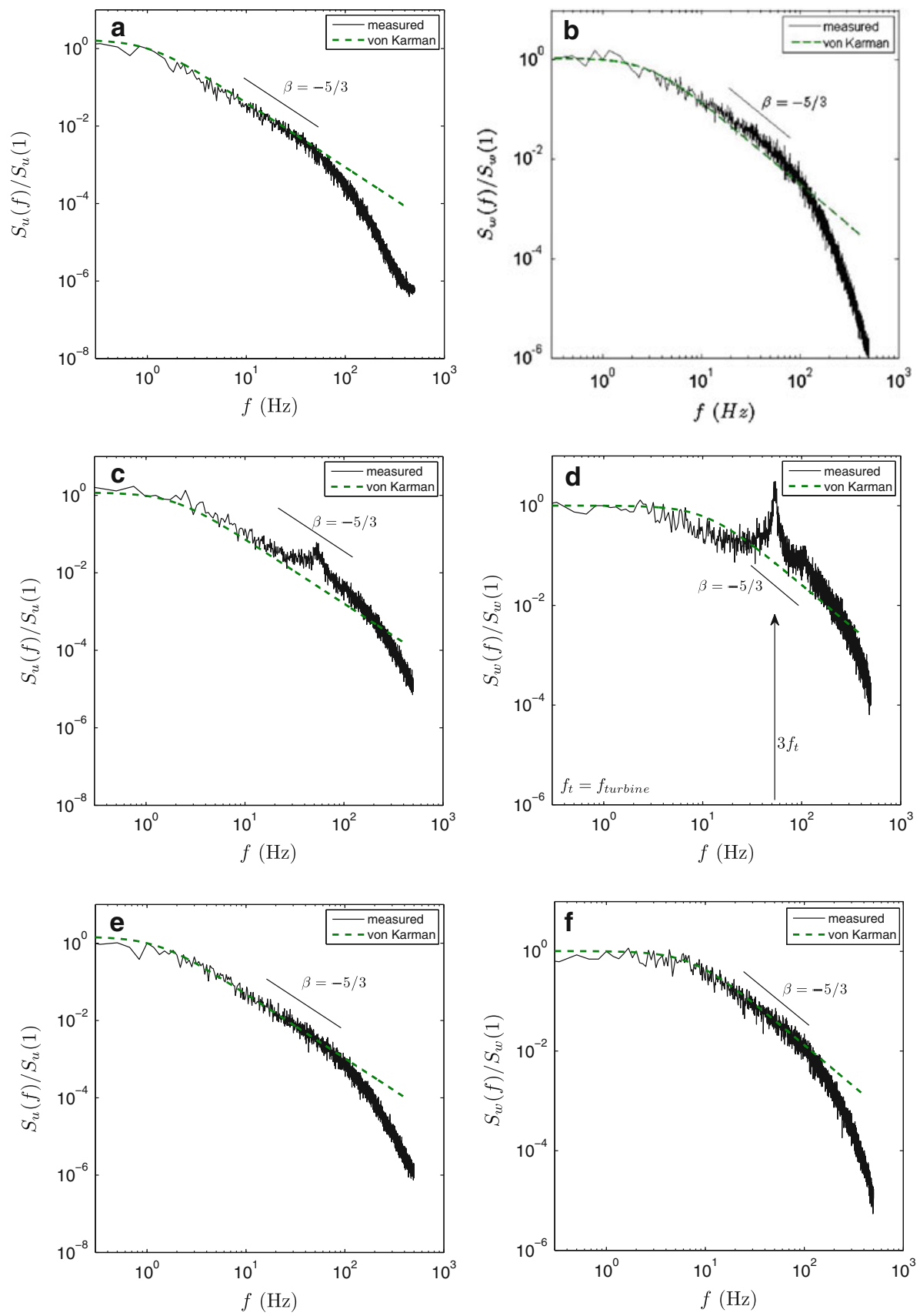

Fig. 12 Measured and modelled power spectrum of the streamwise $(u)$ and vertical $(w)$ velocity components in the neutral boundary layer at the turbine top tip height. a $u$-component, incoming flow; $\mathbf{b} w$-component, incoming flow; $\mathbf{c} x / d=1, u$-component; $\mathbf{d} x / d=1, w$-component; $\mathbf{e} x / d=5, u$-component; and $\mathbf{f} x / d=5$, $w$-component 


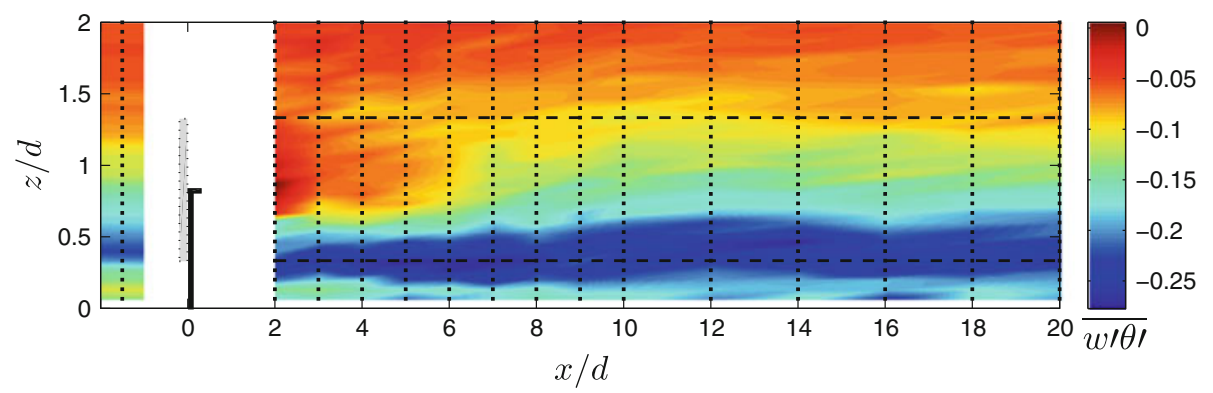

Fig. 13 Non-dimensional distribution of turbulent heat flux at different locations downwind of the turbine (at zero span) in the stable boundary layer. Horizontal dashed lines represent the turbine bottom and top tip heights and dots indicate measurement locations

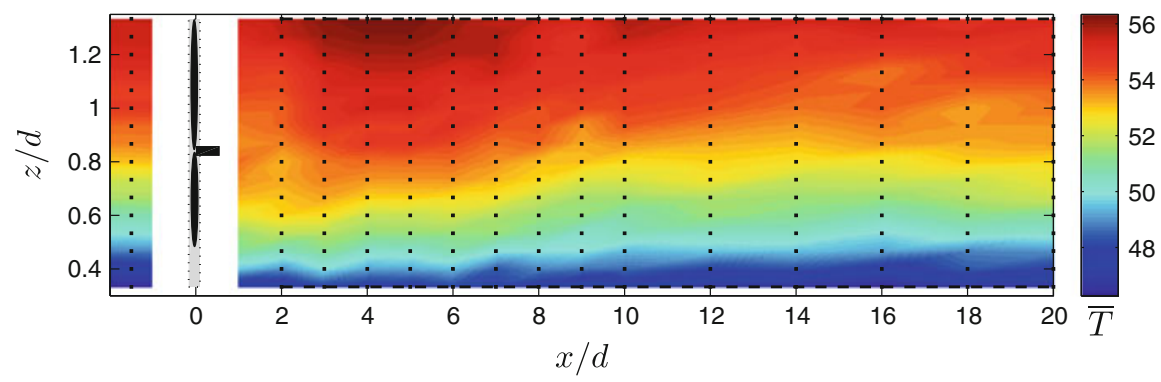

Fig. 14 Mean temperature distribution $\left({ }^{\circ} \mathrm{C}\right.$ ) at different locations downwind of the turbine (at zero span) in the stable boundary layer. Horizontal dashed lines represent the turbine bottom and top tip heights and dots indicate measurement locations

Wake instability, associated with meandering, can be detected in the low frequency components of the measured velocity in both thermal stratification cases. The characteristic frequency of this large-scale meandering motion is found to be lower in stable stratification by roughly $18 \%$ with respect to that found in the neutral case. Figure 15 shows the radial (vertical) low frequency velocity spectrum, at the turbine hub-height and $x / d=1$, for the two thermal stratification cases (there a Butterworth low-pass filter with a cut-off frequency of $15 \mathrm{~Hz}$ was applied). From that Figure, the predominant frequencies associated with the meandering motion are $f_{m} \approx 6.7$ and $f_{m}=5.5 \mathrm{~Hz}$ for the neutral and stable boundarylayer cases respectively, with characteristic secondary motions measured up to a distance of $x / d \approx 4$ (results not shown here). In general, these motions are affected by the turbine thrust coefficient $\left(C_{T}\right)$ and tip speed ratio $(\lambda)$ as shown by Medici and Alfredsson (2008), who detected them (at frequencies around $10 \mathrm{~Hz}$ ) in wind-tunnel experiments at $x / d=1$ behind a miniature turbine in a boundary-layer flow.

\section{Summary}

Wind-tunnel experiments were carried out to study turbulent statistics in the wake of a model wind turbine placed in a boundary-layer flow under both thermally neutral and stably stratified conditions. A customized 3-wire anemometry (cross-wire and cold wire) was used to obtain high resolution and simultaneous measurements of temperature and two velocity 

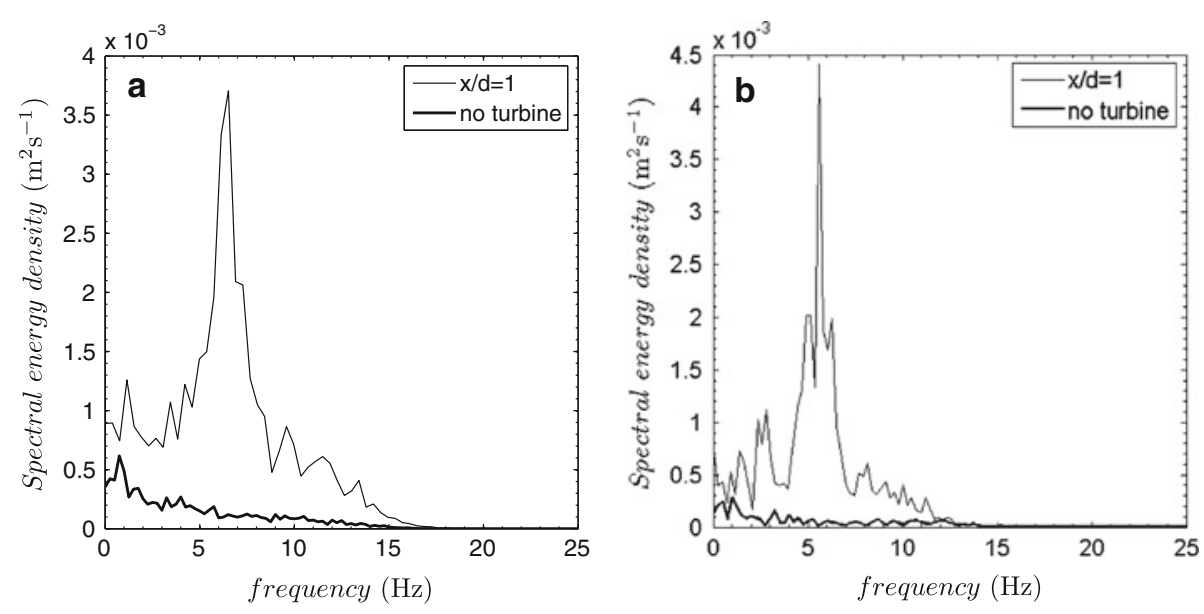

Fig. 15 Power spectrum of the low frequency components of the radial velocity at the turbine hub height and $x / d=1$ : a neutral boundary layer, $\mathbf{b}$ stable boundary layer

components (streamwise and vertical) at different locations behind the wind turbine. Flow statistics (mean velocity, turbulence intensity, kinematic shear stress) were calculated in the two thermal stratification scenarios for comparison and spatial characterization $(x-z$ plane at zero span). The kinematic heat flux and mean temperature distributions were also calculated in the stable case.

Results show a clear effect of the turbine wake on the turbulence statistics as far as 20 rotor diameters downwind of the turbine. The velocity deficit is found to have a nearly axisymmetric shape, which can be approximated by a Gaussian distribution in the directions perpendicular to the turbine axis. The maximum deficit is located at the hub height and follows a power-law decay with distance from the turbine. The rate of decay is found to be higher in the stable stratified case. This simple behaviour of the velocity deficit could be used to develop (or extend existing) simple wake velocity formulations, based on similarity arguments and conservation of momentum, to account for the distortion of the mean velocity profile in the wake of wind turbines placed in boundary-layer flows.

Due to the non-uniform distributions of the incoming mean velocity and velocity fluctuations, the turbulence intensity does not show axisymmetric behaviour, and is found to be stronger above the hub height and around the turbine tip. The enhancement of turbulence intensity is associated with strong shear and turbulence kinetic energy production above the hub height. Due to boundary-layer effects (relative higher incoming turbulence intensity and shear), negligible effects are observed near the bottom tip. In addition to the boundary-layer effects, negative buoyancy acting on the stable case precludes an enhancement of turbulence intensity in the lower part of the wake. Maximum turbulence intensity in the neutral case is found to be located at a distance of about 4-5.5 rotor diameters, which are common separations between wind turbines in wind farms. In the stable case, the stronger shear in the incoming flow produces a slightly greater turbulence intensity above the hub height, which extends between 3 and 6 rotor diameters downwind of the turbine location.

Power spectra of streamwise and vertical velocity components show strong influence of the tip vortices on the flow at the top tip height at $x / d=1$, with stronger effects in the vertical velocity component. Our analysis also shows the suitability of the von Kármán power spectrum formulation to represent the structure of the turbulence in regions of the wake where 
tip vortices have no important effects. In the stable case, the power spectrum of temperature fluctuations does not show a clear effect of the tip vortices.

Evidence of wake meandering is detected in the low-frequency range of the velocity power spectrum for the two stratification cases. The characteristic frequency of this largescale motion is found to be lower in the stable case by roughly $18 \%$ with respect to that found in the neutral case. Specifically, predominant peaks at $f_{m} \approx 6.7$ and $f_{m} \approx 5.5 \mathrm{~Hz}$ are found in the neutral and stables scenarios, respectively. This meandering is evident up to a distance of $x / d=4$.

Future research will focus on studying the effects of different thermal stratification conditions (including unstable conditions) on the structure of wind turbine wakes. In addition, wind-tunnel experiments will be performed to better understand turbulent boundary-layer flow inside and above wind farms for different farm layout configurations and different thermal stratification conditions.

Acknowledgements The authors gratefully acknowledge the assistance of James Tucker during the course of the experiments. Funding was provided by NSF (grant EAR-0537856), NASA (grant NNG06GE256) and Xcel Energy through the Renewable Development Fund (grant RD3-42). Computing resources were provided by the University of Minnesota Supercomputing Institute.

\section{References}

Archer C, Jacobson M (2003) Spatial and temporal distributions of U.S. winds and wind power at $80 \mathrm{~m}$ derived from measurements. J Geophys Res 108:1-20

Arya S (1968) Structure of stable stratified turbulent boundary layer. Technical Rept. No. 0868676, Colorado State University, $176 \mathrm{pp}$

Arya S (2001) Introduction to micrometeorology, 1st edn. Academic Press, New York, 420 pp

Barthelmie R, Larsen G, Pryor S, Jorgensen H, Bergstrom H, Schlez W, Rados K, Lange B, Volund P, Neckelmann S, Mogensen S, Schepers G, Hegberg T, Folkerts L, Magnusson M (2004) ENDOW (efficient development of offshore wind farms): modelling wake and boundary layer interactions. Wind Energy 7:225-245

Bruun HH (1995) Hotwire anemometry, principles and signal analysis. Oxford University Press, UK, 507 pp

Carper M, Porté-Agel F (2008) Subfilter-scale fluxes over a surface roughness transition. Part I: Measured fluxes and energy transfer rates. Boundary-Layer Meteorol 126:157-179

Chamorro LP, Porté-Agel F (2009) A wind-tunnel investigation of wind-turbine wakes: boundary-layer turbulence effects. Boundary-Layer Meteorol 132:129-149

Chaudhry F, Meroney R (1973) A laboratory study of difussion in stably stratified flow. Atmos Environ 7:443454

Derbyshire S (1990) Nieuwstadt's stable boundary layer revisited. Q J Roy Meteorol Soc 116:127-158

Frandsen S, Barthelmie R, Pryor S, Rathmann O, Larsen S, Hojstrup J (2006) Analytical modelling of wind speed deficit in large offshore wind farms. Wind Energy 9:39-53

Hunt J, Stretch D, Britter R (1988) Length scales in stably stratified turbulent flows and their use in turbulence models. In: Puttock JS (ed) Stably stratified flows and dense gas dispersion. Clarendon Press, Oxford, pp 285-321

Jensen N (1983) A note on wind turbine interaction. Riso-M-2411, Risoe National Laboratory, Roskilde, Denmark, $16 \mathrm{pp}$

Katic I (1986) A simple model for cluster efficiency. In: Proceedings of the European wind energy association conference and exhibition, Rome, Italy, pp 407-409

Larsen G, Madsen H, Bingol F, Mann J, Ott S, Sorensen J, Okulov V, Troldborg N, Nielsen M, Thomsen K, Larsen T, Mikkelsen R (2007) Dynamic wake meandering modeling. Riso-R-1607, Risoe National Laboratory, Roskilde, Denmark, $83 \mathrm{pp}$

Magnusson M, Smedman A (1999) Air flow behind wind turbines. J Wind Eng Ind Aerodyn 80:169-189

Mahrt L (1998) Stratified atmospheric boundary layer and breakdown of models. Theor Comput Fluid Dyn 11:263-279

Medici D, Alfredsson P (2008) Measurement behind model wind turbines: further evidence of wake meandering. Wind Energy 11:211-217 
Morfiadakis E, Glinou G, Koulouvari M (1996) The suitability of the von Karman spectrum for the structure of turbulence in a complex terrain wind farm. J Wind Eng 62:237-257

Ohya Y, Neff D, Meroney R (1997) Turbulence structure in a stratified boundary layer under stable conditions. Boundary-Layer Meteorol 83:139-161

Ohya Y, Nakamura R, Uchida T (2008) Intermittent bursting of turbulence in a stable boundary layer with low-level jet. Boundary-Layer Meteorol 126:349-363

Okulov V, Sorensen J (2007) Stability of helical tip vortices in a rotor far wake. J Fluid Mech 576:1-25

Pérez I, García M, Sánchez M, De Torre B (2005) Analysis and parameterisation of wind profiles in the low atmosphere. Solar Energy 78:809-821

Rosen A, Sheinman Y (1996) The power fluctuations of a wind turbine. J Wind Eng Ind Aerodyn 59:51-68

Schlichting H (1968) Boundary layer theory, 6th edn. McGraw-Hill, New York, $817 \mathrm{pp}$

Smedman A, Högström U, Bergström H (1996) Low level jets—a decisive factor for off-shore wind energy siting in the Baltic Sea. J Wind Eng 20:137-147

Stull R (1988) An introduction to boundary layer meteorology. Kluwer Academic Publishers, Dordrecht, $670 \mathrm{pp}$

van der Berg G (2008) Wind turbine power and sound in relation to atmospheric stability. Wind Energy 11:151-169

Vermeer L, Sorensen J, Crespo A (2003) Wind turbine wake aerodynamics. Prog Aerospace Sci 39:467-510

von Kármán T (1948) Progress in the statistical theory of turbulence. Proc Natl Acad Sci USA 34:530-539

Vukoslavcevic P, Wallace J (2002) The simultaneous measurement of velocity and temperature in heated turbulent air flow using thermal anemometry. Meas Sci Technol 13:1615-1624 